\title{
El modelo pedagógico de materiales digitales para Educación Infantil elaborados por editoriales gallegas
}

\author{
The pedagogical model of digital materials for early childhood \\ education developed by Galician publishers
}

RECIBIDO 31/3/2021 ACEPTADO 30/6/2021 PUBLICADO 1/12/2021

\begin{abstract}
D Rosa María Méndez García
Departamento de Pedagogía y Didáctica, Universidade da Coruña, España

rosa.mendez@udc.es

(D) Lucía Casal de la Fuente

Área de Promoción y Proyectos Internacionales, Universidade de Santiago de Compostela, España

lucia.casal@usc.gal
\end{abstract}

(iD) Nerea Rodríguez Regueira

CEIP Canicouva enVigo (Pontevedra), España

nrregueira@edu.xunta.gal

(D) Daniela Gonçalves

Departamento de Formação de Professores, Universidad de Oporto, Portugal

dag@esepf.pt

\section{RESUMEN}

Teniendo en cuenta el carácter innovador que, a priori, implica la incorporación de materiales didácticos digitales al proceso de enseñanza-aprendizaje, y siendo la Educación Infantil una de las etapas del sistema educativo reglado que debiera destacar también por su carácter innovador, entendemos que es de gran interés conocer el modelo pedagógico que subyace a estos materiales. Por lo tanto, el objetivo de este artículo es analizar el modelo pedagógico de materiales para el segundo ciclo de Educación Infantil publicados por editoriales gallegas, como parte de la oferta de recursos educativos digitales a disposición del profesorado en esta comunidad. Concretamente, el análisis se centra en el tipo de actividades y recursos complementarios, así como el rol del profesorado y del alumnado de una muestra de ocho materiales de cuatro editoriales diferentes. Para el análisis de los datos, se tiene en cuenta el grado de coherencia de lo hallado con lo que establece el currículo oficial para esta comunidad autónoma. Los resultados sugieren que, si bien el carácter digital del material no supone cambios significativos en los modelos pedagógicos y no se aprovechan las potencialidades que brindan las tecnologías, el carácter abierto y flexible de esta etapa posibilita la adaptación de los mismos.

PALABRAS CLAVE material didáctico, medios audiovisuales, modelo didáctico, educación de la primera infancia, industria editorial. 


\section{ABSTRACT}

Considering the innovative nature which, a priori, implies the use of didactic digital materials in the teaching-learning process, and being early childhood education one of the stages of the regulated educational system that should stand out also by its innovative character, it is of great interest to know the underlying pedagogical model of these materials. Therefore, the aim of this piece of research is to analyse the pedagogical model of materials for the second cycle of early childhood education published by Galician publishers, as part of the supply of digital educational resources at the disposal of the teachers of this region. Specifically, the analysis is focused on the type of activities and complementary resources, and the roles assigned to teachers and children, being the sample a total of eight materials designed by four different publishers. For the data analysis it is considered the degree of coherence between the findings and what the official curriculum stablishes for this autonomous region. The findings suggest that although the digital constitution of the material does not entail significative changes in the pedagogical models, and that the technological potential is not seized, the open and flexible nature of this stage makes it possible to adapt the materials.

KEYWORDS instructional materials, multimedia materials, teaching models, early childhood education, publishing industry.

\section{INTRODUCCIÓN}

La implementación de las tecnologías en la escuela de forma efectiva fue y sigue siendo un reto, sobretodo si consideramos la vorágine de cambios que conlleva la digitalización y la hiperconexión actual. Igualmente, parece indudable su potencial para el cambio educativo, tal y como han señalado, entre otros, Ricaurte y Carli (2016), quienes apuntan a que la introducción de las tecnologías digitales podría ayudar a superar enfoques predominantemente tradicionales; o Selwyn (2016), que sitúa su potencial en un rango que fluye desde el mero mejoramiento de los procesos y resultados de aprendizaje hasta la revolución educativa total.

Dentro de este contexto está cada vez más extendido el uso de los materiales didácticos digitales (MDD¹), entendidos como "un paquete estructurado didácticamente de objetos digitales en línea dirigidos a facilitar al alumnado el desarrollo de experiencias de aprendizaje entorno a una unidad de saber o competencia" (Area, 2017, pp. 4-5). Sin embargo, tal y como señala Rodríguez (2019), su uso debe llevar paralelamente una reflexión sobre aquellos aspectos que dan respuestas a las necesidades educativas propias de una sociedad digital como son, entre otros, los señalados por Area (2017): nuevos escenarios de aprendizaje, los nuevos roles de los agentes educativos o los cambios en la producción, distribución y consumo. Es preciso señalar que "la utilización de recursos materiales durante la primera escolarización en la educación formal se realiza, en gran medida, de manera rutinaria y con una fuerte dependencia de las editoriales" (Moreno, 2017, p. 19). Esta dependencia tiene como resultado la subordinación de los docentes y, por extensión de los centros educativos, al proyecto pedagógico diseñado por las editoriales. Este hecho se ha constatado ampliamente, sobretodo en lo que al libro de texto se refiere, por autores como Bonafé (2002), Gimeno (1998) o Rodríguez (2000), entre otros.

Son múltiples los estudios que han abordado la utilización e integración de los MDD en el contexto español (Losada, \& Rodríguez, 2019; Sanabria et al., 2017), y han coincidido en señalar que los MDD crea-

1 De aquí en adelante, por economía del lenguaje, usaremos MDD para referirnos a los materiales didácticos digitales. 
dos por las editoriales, sobre todo haciendo referencia a los libros de texto digitales en los niveles de la educación primaria y secundaria, no suponen cambios significativos respecto a los materiales didácticos en papel, sino que en la mayoría de los casos son meras digitalizaciones enriquecidas con algunos recursos multimedia (Area, 2019; Area, \& Rodríguez, 2017). Asimismo, se ha constatado que la oferta de MDD es cada vez más amplia, abarcando más niveles educativos, formatos e integrando nuevas potencialidades como la inteligencia artificial (Gabarda et al., 2021; Peirats et al. 2016).

En la etapa de educación infantil $\left(\mathrm{EI}^{2}\right)$, contexto en el que se centra la presente investigación, abordar el estudio de los MDD resulta controvertido por variantes tan diversas como la variedad de materiales que se utilizan, la variedad de enfoques pedagógicos en los que se puede situar el profesorado, las características psicoevolutivas del alumnado que desaconsejan los tiempos prolongados ante las pantallas o la no consideración de esta etapa dentro de las políticas para la integración de las TIC en las aulas, entre otros. Estas pueden ser algunas de las razones que justifiquen la falta de investigaciones en torno a esta temática. Ahora bien, la relevancia de la misma torna incuestionable cuando el propio currículo de El en la Comunidad Autónoma de Galicia establece como uno de los objetivos de la etapa, que empezará a trabajarse desde el primer ciclo, "Acercarse, en la medida de sus posibilidades, al uso de las tecnologías de la información y de la comunicación" (Decreto 330/2009, p. 10774). La El en España fue progresando durante el siglo XX de manera incierta. El aire fresco de sucesivas corrientes de innovación pedagógica como el movimiento Escuela Nueva, las escuelas freinetianas, montessorianas y decrolinianas, la Institución Libre de Enseñanza, o los Movimientos de Renovación Pedagógica resultaron ser determinantes para inclinar la balanza en esta etapa educativa hacia modelos pedagógicos activos (Zabalza, \& Zabalza, 2012). La capacidad del profesorado para identificar o crear contextos y recursos estimulantes donde el alumnado pueda ir desarrollándose de una forma activa, equilibrada, libre, feliz y en contacto con la naturaleza, así como para asumir un rol de guía o acompañante más que de transmisor de un contenido preestablecido propio de modelos pedagógicos más convencionales, es la apuesta que prevalece y que sigue luchando por hacerse sitio en las aulas de infantil iniciado el siglo XXI.

Concretamente, el modelo pedagógico que se establece en el currículo oficial gallego (Decreto 330/2009) alude a un enfoque globalizador adoptando las secuencias de aprendizaje, los proyectos o las unidades didácticas como opciones metodológicas siempre que sean capaces de abordar contenidos de diferente tipo y de distintas áreas. Sin descartar la importancia de introducir cierto tipo de tareas específicas dirigidas al afianzamiento de habilidades o destrezas parciales dentro del marco integral del desarrollo personal, se insiste en la pertinencia de la introducción de actividades globales a partir de la configuración de situaciones problemáticas significativas para el alumnado, tanto de naturaleza personal como social. La participación activa y la interacción durante el proceso educativo se establecen como ejes fundamentales, y la asamblea, la pregunta abierta y el juego se identifican como actividades de gran ayuda ante tal fin. Desde el punto de vista organizativo, se pone en valor la coexistencia de diferentes formas de agrupamiento durante el desarrollo de las distintas propuestas didácticas (gran grupo, grupo clase, pequeño grupo y pareja), sin descartar

2 De aquí en adelante, por economía del lenguaje, usaremos El para referirnos a la educación infantil. 
la pertinencia de ciertos momentos de actividad individual. Dentro de este modelo pedagógico, el rol que se dibuja para el profesorado se articula en torno a las siguientes premisas:

- Potenciar el desarrollo de habilidades de pensamiento que ayuden al alumnado a encontrarle sentido a su experiencia (comprender), para lo que es imprescindible partir de sus esquemas de conocimiento previos.

- Mediar entre el alumnado y la cultura, dirigiendo, sugiriendo y, especialmente, acompañando, guiando, ayudando, animando, y abriendo caminos.

- Crear ambientes de aprendizaje garantes de la libertad de expresión (derecho fundamental), que estimulen la participación y el reconocimiento de la diversidad, que pongan en valor la diferencia y que no coarten la creatividad.

Como ha sido mencionado anteriormente, al alumnado se le presupone un rol activo durante todo el proceso de enseñanza aprendizaje, otorgándole la responsabilidad de proponer, organizar y gestionar sus actividades y materiales, en el marco de los retos que se consensua resolver, así como participar activamente en procesos de autoevaluación y coevaluación de su propio aprendizaje.

Este modelo pedagógico necesita medios y recursos cómplices, que faciliten la exploración activa, que permitan el acceso a nuevos objetos o fuentes de conocimiento, que estimulen la comprensión y la creatividad, que potencien la interacción y que apuesten por la incorporación de experiencias globalizadoras y significativas para los niños y niñas como ejes facilitadores del desarrollo integral. El propio Decreto 330/2009 describe también los materiales, que deben ser lo suficientemente versátiles como para ser capaces de adaptarse a los tipos de contenido, a las necesidades del contexto y a la individualidad del alumnado; lo suficientemente diversos como para permitir distintos grados de uso; y lo suficientemente significativos para despertar el interés por la exploración y experimentación.

Tal y como hemos avanzado, la finalidad de este artículo es determinar el modelo pedagógico que subyace a los MDD que componen la muestra de nuestro estudio y si estos suponen un cambio significativo con respecto de los materiales empleados habitualmente en las aulas gallegas. Por ello, consideramos fundamental analizar que dice al respecto el currículo de esta comunidad autónoma.

Como hemos visto, nos encontramos en una realidad caracterizada por la inevitable digitalización de los centros educativos (más aun a partir del año 2020 debido a la crisis sanitaria) y por la influencia que ejercen las editoriales sobre las escuelas a través de los materiales que publican. A la vista de la escasez de estudios existentes sobre materiales digitales para la etapa de El, se hace necesario investigar en dicho campo, dada la relevancia que cada vez más el imperativo tecnológico adquiere en el mundo educativo. Por ello, concretamos el objetivo de este artículo en descubrir cuál es el modelo pedagógico de los materiales diseñados por editoriales gallegas para $2 .^{\circ}$ ciclo de El.

\section{DISEÑO Y METODOLOGÍA}

\subsection{Características de la muestra}

En el marco del proyecto "Los materiales didácticos digitales en la Educación Infantil. Análisis y propuestas para su uso en la escuela y el hogar (RTI2018-093397-B-100)", en el que participa personal docente e investigador de universidades de tres comunidades autónomas españolas (La Laguna, Santiago de Compostela, 
Coruña y Valencia), el equipo de la Universidad de Santiago de Compostela analizó un total de 58 plataformas editoriales, tanto estatales como gallegas: 37 de ellas ofrecían materiales dirigidos a la etapa de El y 19 contaban con plataforma digital. Estamos ante un muestreo por conveniencia en el que se seleccionaron en primer lugar 4 editoriales atendiendo a estos criterios:

- Reconocimiento de las editoriales a nivel gallego por su elevada presencia en las aulas de El.

- Abordaje de los tres niveles del segundo ciclo de El y de diferentes áreas curriculares.

- Edición del material prioritariamente en lengua gallega, aunque se incluyó también material publicado en lengua castellana.

- Tipología del material diversa (libros de texto digitales, álbumes ilustrados, juegos y vídeos didácticos, etc.).

- Posibilidad de acceso.

Tras observar que en cada editorial se daba una consistencia en la tipología de materiales organizándose en colecciones, se seleccionaron para el análisis dos materiales de cada una de ellas, resultando una muestra de 8 materiales respetando el criterio de diversidad temática, de niveles y de tipo de material.

En la siguiente tabla se muestran los materiales objeto de análisis atendiendo a su tipología, formato y finalidad (lúdica, educativa u otras).

TABLA 1. Características de la muestra

\begin{tabular}{cccc}
\hline Material & Tipo & Formato & Finalidad \\
\hline M1 & ODA & Juego didáctico & Lúdico-educativa \\
\hline M2 & MDD & Repositorio de 6 propuestas didácticas & Educativa \\
\hline M3 & MDD & Vídeo didáctico & Lúdico-educativa \\
\hline M4 & ODA & Álbum ilustrado digital & Lúdico-educativa \\
\hline M5 & ODA & Álbum ilustrado digital & Divulgativa \\
\hline M6 & ODA & Vídeo & Educativa \\
\hline M7 & MDD & Método de lectoescritura (2 cuadernos, 1 mural y 70 fichas) & Educativa \\
\hline M8 & MDD & Método de lectoescritura &
\end{tabular}

Se trata de 8 materiales que, en función de su tipología, podemos describir de la siguiente manera:

- M1: juego didáctico interactivo sobre los animales de la granja.

- M2: repositorio de material didáctico situado en una página web que ofrece 6 recursos didácticos sobre: transmisión de valores patrimoniales, hábitos de salud y responsabilidad viaria o relacionada con las mascotas.

- M3: Serie de vídeos educativos orientados al aprendizaje del inglés.

- M4 y M5: álbumes ilustrados interactivos.

- M6: vídeo-presentación de un escritor e ilustrador que forma parte de una videoteca cuyas secciones principales son: cuentacuentos, entrevistas noticias, visitas a lugares de interés cultural, tráileres, vídeos musicales, etc.

- M7 y M8: Métodos de lectoescritura compuestos por cuadernos, libro de lecturas, posters y fichas. 


\subsection{Instrumento y procedimiento}

El instrumento usado por el personal investigador para el análisis de los materiales fue diseñado exprofeso para el proyecto en el que se enmarca este trabajo. Dicho instrumento ha sido adaptado, considerando la idiosincrasia de la El, a partir del instrumento empleado en el Proyecto "Los materiales didácticos digitales en la Educación Infantil. Análisis y propuestas para su uso en la escuela y elhogar (RTI2018-093397-B-I00)", cuyas características pueden consultarse en el trabajo de Rodríguez (2019). Se trata de una guía de análisis compuesta por ocho dimensiones, a saber: datos de identificación de la evaluación, datos de identificación del material, estructura del material, dimensión tecnológica, dimensión de diseño, dimensión pedagógica, dimensión de contenido, evaluación y seguimiento, y comentarios finales.

El presente artículo pone su foco de atención en la dimensión pedagógica de los materiales que analizaremos a partir de las tres variables que pasamos a explicar:

1. Tipo de tareas que ofrecen: se analizará el tipo de tareas presentes en cada material en función de tres criterios:

a. El nivel de exigencia cognitiva: de acuerdo con Doyle (1983), Elliot (1990) y Gimeno (1988) definimos:

- Tareas de bajo nivel de exigencia cognitiva, se trata de tareas de carácter específico dirigidas fundamentalmente a la automatización de una rutina o al contacto con una información, pueden.

- Tareas de reconocimiento: implican el reconocimiento o la identificación de la respuesta correcta dentro de una serie de opciones propuestas, no exigiendo ni siquiera la memorización.

- Tareas de memoria o recuerdo: conllevan la reproducción oral o escrita de información adquirida previamente (episódicamente), referida a datos o hechos.

- Tareas de resolución de problemas algorítmicos o de rutina: implican la aplicación de una fórmula, algoritmo o pasos de una rutina que llevan a una determinada respuesta o resultado.

- Tareas de alto nivel de exigencia cognitiva, o también conocidas como actividades globales. Su carácter es más complejo pues implica el desarrollo de varias capacidades, y dentro de ellas caben tareas más específicas.

- Tareas de comprensión reconstructiva: exigen reconocer la información de acuerdo con los términos, conceptos e ideas en los que se presenta, y dar una versión propia de la misma, aunque sin desligarse demasiado de la lógica del discurso planteado.

- Tareas de comprensión reconstructiva global: exigen reconocer la información y dar una versión propia de la misma, aplicando procedimientos de forma contextualizada, extrayendo consecuencias y conclusiones argumentadas.

- Tareas de comprensión constructiva o de descubrimiento: exigen construir nuevos y originales significados que la superen.

b. La finalidad dentro de una secuencia didáctica: iniciación y motivación, explicitación de conocimientos previos, desarrollo de contenidos, ampliación y refuerzo, y evaluación.

c. El agrupamiento del alumnado para su realización: individual, parejas, pequeño grupo, grupo-clase o gran grupo. 
2. Rol que promueven para el profesorado y el alumnado. En este caso se atiende a dos criterios:

a. El rol que se infiere ante el proceso de enseñanza-aprendizaje para ambos protagonistas, que se interpreta desde la actividad a la pasividad desde el punto de vista del alumnado, y desde la guía o acompañamiento a la dirección o transmisión desde el punto de vista del profesorado.

b. Las posibilidades de modificación, adaptación o cambio que ofrece o sugiere el propio material, tanto para el profesorado como para el alumnado, que se interpretan desde el polo de la autonomía al polo de la dependencia.

3. Tipo de recursos complementarios que incluyen, de ser el caso, y cómo estos contribuyen a afianzar el modelo pedagógico. Se entiende por recursos complementarios la variedad de materiales, que no necesariamente han sido creados con una finalidad didáctica y que apoyan y acompañan al material didáctico básico que se usa para tratar un contenido o materia concreta (Liébana et al., 2001). Dentro de estos materiales, podemos encontrar una amplia amalgama que podríamos clasificar entre materiales creados con un fin didáctico y los que, en un momento dado, pudieran ser empleados con este fin. En este último caso, por ejemplo, podríamos situar, tal y como señala Area (1994), los periódicos, revistas o folletos. Estos recursos complementarios pueden dirigirse al alumnado, al profesorado y/o a las familias. Los materiales complementarios para el profesorado son:

Aquellos documentos que acompañan o son complementarios de materiales dirigidos al alumnado (por ejemplo, la documentación o ficha de un vídeo didáctico, la guía del profesor de un libro de texto, el folleto explicativo de un paquete multimedia, etc.). La función de esta documentación consiste en explicar al profesorado las características y modos de uso didáctico del material del alumno (Area, 1994, p. 11).

Esta conceptualización de recursos complementarios resulta, si cabe, más amplia en el nivel de El donde "la acción educativa puede llevarse a cabo con cualquier material" (Cañas, 2010, p. 1). Si atendemos, por ejemplo, a la Teoría de las Piezas Sueltas (Daly, \& Beloglovsky, 2016; Nicholson, 1973) cualquier objeto puede ser utilizado como un material complementario en el aula (una piedra, un palo, un tique, un trozo de plástico, etc.). En este sentido, resulta imprescindible que el profesorado de este nivel educativo reflexione sobre su propia práctica seleccionando aquellos medios que mejor se adapten a cada situación concreta.

Analizar el tipo de recursos complementarios que acompañan a los materiales didácticos seleccionados es, pues, una buena forma para determinar el tipo de modelo pedagógico que subyace a los mismos. De este modo, si el material está pensado como recurso único, es previsible que no incluya ningún tipo de complemento. Sin embargo, si el material está pensado considerando, por ejemplo, el Diseño Universal para el Aprendizaje (DUA), es previsible que incluya recursos diversos para atender a la diversidad del aula (Alba, 2012; Martínez, \& Fernández, 2020; Sánchez, \& Arathoon, 2017).

\section{RESULTADOS}

El apartado resultados se divide en tres subapartados, en buena lógica con el número de variables estudiadas. En cada uno de los siguientes tres subapartados, indicaremos entre paréntesis el número de materiales 
analizados del total $(n=8)$ que coinciden con la característica que se describe. ${ }^{3}$ También se incluirán entre comillas los comentarios escritos en cada una de las fichas de análisis de los materiales que el equipo investigador destacó y que contribuyen a enriquecer el discurso. En este caso, a su lado se identificará la ficha del material de la cual han sido extraídos ${ }^{4}$.

\subsection{Tipo de tareas de los materiales}

Lo primero que debemos señalar sobre los tipos de actividades es que las tareas de reconocimiento están presentes en 3 de los 8 materiales, ya sea a través de tareas de relacionar conceptos (M2), unir con flechas y completar (M7), o elegir una opción entre varias propuestas (M8).

Por su parte, las tareas de memoria ocupan una parte importante en 7 de los 8 materiales, precisamente aquellos que tienen una intencionalidad educativa explícita o incluso prioritaria frente a la lúdica. Pueden centrarse en ejercitar la memoria a corto plazo, por ejemplo, con juegos de memoria donde la tarea sea encontrar parejas (M4), o pueden exigir el recuerdo de información adquirida previamente, tal y como recoge el siguiente ejemplo recuperado de una ficha de análisis:

\footnotetext{
"La actividad que prevalece es una actividad de presentación del vocabulario y las sentencias básicas asociadas en el idioma inglés, que se repite dos o tres veces a través de la intervención de los distintos personajes, para afianzar su adquisición, con apoyo visual sobre cada término que suele aparecer escrito en inglés en la pantalla" (M3).
}

En cuanto a las tareas de resolución de problemas algorítmicos y de rutina, debemos destacar que aparecen en 4 de los 8 materiales y que son de diferente índole: hojas de registro para sistematizar acciones y desarrollar hábitos y rutinas (M2), puzles y juegos de ordenar series (M5) y rutinas de grafomotricidad (M7 y M8).

A pesar de que esto es lo que prevalece, avanzamos que al menos en un par de materiales hemos encontrado actividades abiertas que favorecen la creatividad, a las que nos referiremos más adelante.

Las tareas de comprensión reconstructiva están presentes en 2 de los 8 materiales, siendo este nivel de exigencia cognitiva el más elevado que promueven. Se trata de los M7 y M8 en los que se alude a la comprensión lectora de la siguiente manera:

"finaliza con diversas actividades orientadas a la comprensión lectora, usando palabras que llevan la letra estudiada en cada unidad" (M7).

Las tareas de comprensión constructiva o de descubrimiento se han constatado en 3 de los 8 materiales, entendiendo el descubrimiento en términos de creatividad.

“permiten desarrollar la motivación y la creatividad (ilustraciones, recetas, recortables, ...), amplían conocimientos (recetas, adivinanzas, ...)" (M2).

No hemos podido identificar tareas de comprensión reconstructiva global en ninguno de los materiales,

3 Por ejemplo, si se señala (2/8), querrá decir que tal característica la cumplen 2 materiales de un total de 8, la muestra de este estudio.

4 Por ejemplo, si se señala (M5), querrá decir que tal extracto pertenece a la ficha de análisis del material número 5. 
si bien el nivel de exigencia cognitiva que fomentan está garantizado en los materiales que hemos presentado en la última categoría de tareas.

A la vista de lo expuesto, parece que el conjunto de materiales examinados evidencia un predominio de actividades de bajo nivel de exigencia cognitiva, especialmente de memoria (7/8), aunque también de rutina (4/8) e incluso de reconocimiento (3/8). Son tan solo 2 dentro del total los que incluyen algunas tareas de comprensión, y tan solo 3 los que incorporan tareas abiertas que tratan de promover el descubrimiento y la creatividad.

En términos absolutos, que 5 de los 9 materiales incluyan tareas de alto nivel de exigencia cognitiva no parece un mal resultado, el matiz se encuentra cuando, de estos 5, tan sólo 3 han sido elaborados con un enfoque didáctico explícito (M2, M7 y M8), siendo los dos restantes álbumes ilustrados interactivos (M4 y M5), cuya potencialidad didáctica queda en gran medida en el uso que el docente haga de ellos. Asimismo, hay que tener en cuenta que, de estos 3 materiales elaborados con un enfoque didáctico explícito, tan solo 2 (M7 y M8 se corresponden a un producto editorial tradicionalmente elaborado para uso escolar, y que si los analizamos en su conjunto observamos que la presencia de tareas de memoria, rutina y reconocimiento (por este orden), prevalece sobre la presencia de tareas de comprensión, nivel de exigencia máximo que promovían.

Si atendemos a la tipología de tareas en función de su finalidad dentro de una secuencia didáctica, podemos decir que, de los 8 materiales, son 4 los que incluyen tareas diferenciadas a este nivel, concretamente los identificados como MDD. En todos ellos la parte fundamental la ocupan las actividades de desarrollo de los contenidos, que suelen seguir una secuencia lógica y ordenada desde lo sencillo a lo complejo, o intercalando exposición teórica (letra e ilustración) con ejercicios de aplicación. Y excepto uno de ellos (M2), que solo ofrece este tipo de actividades, el resto (3/8) incluyen:

- Actividades de iniciación y motivación al principio de cada sección del material o al principio del propio material.

- Actividades de ampliación y refuerzo sobre lo aprendido.

- Actividades de evaluación o autoevaluación en forma de memoria o de reconocimiento de las tareas realizadas en su totalidad.

Así las cosas, los materiales aludidos presentan, en términos generales, una secuencia didáctica bastante completa. Tan solo se echan en falta las tareas de explicitación de conocimientos previos, tan importantes para favorecer el desarrollo del aprendizaje significativo, que no constan en ninguno de los materiales analizados.

La funcionalidad didáctica de los 4 materiales restantes, todos ellos ODA, dependerá del uso que el profesorado haga de ellos, pero se presuponen con una intencionalidad fundamentalmente motivadora, o de repaso, refuerzo, ampliación, o incluso evaluación.

Para finalizar, ninguno de los materiales explicita en el enunciado de sus tareas si estas son para ser desarrolladas de manera individual o grupal. Ahora bien, teniendo en cuenta que el tipo de actividades predominantes son de bajo nivel de exigencia cognitiva debemos pensar que su diseño está orientado al repaso, refuerzo y memoria individualizada. Por lo tanto, el hecho de que se desarrollen en grupo dependerá fundamentalmente del planteamiento docente. 


\subsection{Tipo de roles que se infieren de los materiales}

Hemos analizado los tipos de roles atendiendo a dos categorías fundamentales: los adoptados por el profesorado y los adoptados por el alumnado.

Comenzaremos por los diferentes roles docentes que promueven los materiales objeto de estudio.

Primeramente, cabe señalar que la inmensa mayoría de los materiales inducen un rol docente "poco activo" o pasivo (6/8), ya que no permiten la edición o modificación del material, no impulsan la interacción entre las diferentes personas participantes ni tampoco el papel de la familia en la producción y uso del mismo. Aquí un extracto de una ficha de análisis:

\footnotetext{
"Tampoco promueve el impulso de las familias en la producción del material, aunque sí es posible que lo usen en casa porque es de fácil aplicación" (M8).
}

No obstante, algunos materiales promueven roles activos en el profesorado (2/8). Aquí mostramos algunos motivos:

- El material permite la interacción entre profesorado/familia y alumnado (2/8), "porque no es un material de uso autónomo para niños y niñas" (M2), por lo que el profesorado debe realizar un esfuerzo de explicación, guía, etc.

- El material es “adaptable a los diferentes tipos de agrupamientos" (M5), por lo que el profesorado puede tomar decisiones metodológicas, por ejemplo.

Dentro de este rol activo, los materiales atribuyen al profesorado funciones como:

- Ayudar a entender la actividad, supervisar e intervenir en las actividades - de ser necesario- (4/8), especialmente por el nivel de lectoescritura que el material requiere para ser trabajado (2/8): se hace necesario su papel de "guía para que las actividades cobren sentido educativo" (M4).

- Transmitir los aprendizajes a desarrollar (M2).

En un caso se da un modelo mixto, la combinación del rol pasivo con rol activo, atribuidos ambos al profesorado. Se indica en la ficha del mismo que el profesorado asume un "rol totalmente pasivo con respecto al recurso (lo adopta) y un rol activo ante el proceso de enseñanza aprendizaje, pues lo dirige según las consignas del propio material. No hay invitación a la opcionalidad, a la realización de propuestas, a la ampliación o contextualización" (M7).

A continuación, expondremos los diferentes roles discentes que los materiales atribuyen al alumnado, atendiendo a lo que se les solicita hacer.

Debemos empezar destacando que, mayoritariamente, los roles discentes son "poco activos" o pasivos (5/8) porque el material:

- No promueve la planificación del propio aprendizaje (5/8).

- No impulsa la interacción entre las diferentes personas participantes (3/8) "más allá de que el profesor explique la tarea y el alumno la realice" (M7).

- No promueve el papel de la familia en la producción y uso del material (4/8), donde el alumnado pudiera servir de guía a la familia, por ejemplo. 
- "Se otorga un rol eminentemente pasivo al espectador" (M3), ya que se trata de un producto audiovisual.

- Está enfocado desde una "estrategia de carácter expositivo, dirigida a la iniciación de la competencia lingüística en el idioma inglés, incidiendo fundamentalmente en la adquisición de vocabulario y pequeñas expresiones, fundamentalmente a nivel de pronunciación y en cierta medida a nivel de escritura" (M3).

- Se centra en el trabajo individual, no favoreciendo el trabajo cooperativo (7/8). Aquí un ejemplo de una ficha de análisis individual:

“En algunas de las secciones el trabajo podría ser en pequeño grupo, pero en su mayoría se dirige a un trabajo individual” (M4).

Otros argumentos que apoyan el rol pasivo del alumnado son:

“[El alumnado] no toma ninguna decisión, sino que su función es realizar los ejercicios y tareas que se van indicando en las sucesivas páginas del material” (M7).

También encontramos materiales que promueven roles activos, en los que el alumnado adopta un papel protagonista (4/8), aunque en diferentes grados, por motivos como los que señalamos a continuación:

- Se permite el uso de manera autónoma por parte del alumnado $(3 / 8)^{5}$, de modo que el material "puede ser adaptado y transformado" (M6), o permite la edición, modificación o adaptación. Por ejemplo, "se puede grabar audio a la par que se va pasando las páginas del álbum ilustrado" (M5). En otro ejemplo se describe lo siguiente:

“El material permite que el niño/a lo manipule de forma autónoma, que seleccione el capítulo a visionar, que lo active y lo detenga de manera autónoma, que amplíe pantalla, que aumente o disminuya voz, no habiendo más opciones de interacción disponibles a su disposición" (M3).

- Promueve "la creatividad, teniendo el estudiante un papel activo" (M4), pues el material ofrece la posibilidad de crear películas, cómics y dibujos libres. "Es posible realizar actividades de manera libre, sobre todo en el último nivel de esta etapa educativa, pero siempre y cuando exista una explicación previa del adulto" (M4).

Al igual que en el caso de los roles docentes, podemos hablar de algunos materiales que promueven roles discentes pasivos y activos a la vez, lo que podría ser denominado un modelo mixto, aunque con una ligera tendencia al rol pasivo. Este es el caso del M3, M4 y M6.

A continuación, destacaremos algunas singularidades más específicas que creemos importante destacar sobre roles que adoptan otros agentes educativos como la familia. Sobre esta, se destaca que algunos

5 Es necesario aclarar que en un material analizado no computado en este epígrafe se destaca lo siguiente "Así, es necesaria la explicación del adulto sobre cada tarea, pero una vez realizada la explicación, el alumnado puede manejarse de forma autónoma. En este sentido, como hay tareas de carácter rutinario, que se repiten unidad tras unidad, poco a poco el alumnado podría ir incrementando en grado de autonomía a este nivel" (M7). Por lo tanto, se trata de un caso en que, tras la explicación por parte de una persona adulta, el material sí puede ser usado de forma autónoma por el alumnado. 
materiales pueden ser usados en familia, ya que los materiales son de fácil uso y pueden promover aprendizajes en todos sus miembros. No obstante, en algunos casos no se impulsa el uso o producción de materiales en el seno familiar.

\footnotetext{
“Explícitamente no hay nada que haga pensar en este objetivo ${ }^{6}$, ahora bien, es una serie muy apropiada para ver en familia, independientemente de la competencia lingüística en inglés. Todos los miembros de la familia pueden ir alfabetizándose de forma paralela en la adquisición de este idioma" (M3).

"Tampoco impulsa el papel de la familia en la producción y uso del material, si bien su claridad estructural y su lógica organizativa permitiría un fácil uso por parte de la familia" (M7).
}

\subsection{Tipos de recursos complementarios que incluyen los materiales}

Lo primero que cabe señalar respecto a los recursos complementarios, es que únicamente están presentes en 3 de los 8 materiales objeto de estudio (ver Tabla 2). En cuanto a su tipología, podemos decir que se trata de materiales para usar por el alumnado guiado por el docente, es decir, no son materiales de autoexploración o que el discente pueda usar de forma autónoma. En los tres casos, nos encontramos con materiales para reforzar los contenidos tratados previamente en los que prevalecen los recursos listos para imprimir.

En líneas generales, podemos decir que la presencia de otras modalidades de contenido asociadas con el carácter digital de los materiales (vídeos, presentaciones, realidad aumentada, hipervínculos...) son nulos.

Es preciso señalar que la mayoría de los portales de las editoriales incluyen una sección para los docentes en la que se pueden encontrar recursos complementarios variados, aunque estos no estén enlazados o directamente relacionados con un material concreto. Del mismo modo, los materiales analizados pueden ser considerados recursos complementarios en sí mismos, al ser digitalizaciones del material impreso, como ocurre por ejemplo con M7 y M8, pudiendo ser utilizados para hacer explicaciones en la pizarra digital interactiva o en situaciones de enseñanza virtual.

TABLA 2. Características de la muestra

\begin{tabular}{lll}
\hline Material & Tipo & Descripción \\
\hline M3 & Material audiovisual & Vídeos de repaso de vocabulario y vídeos de canciones sobre los contenidos \\
\cline { 2 - 3 } & Cuestionario interactivo & $\begin{array}{l}\text { Concurso en el que se opta a ganar una bicicleta respondiendo a una serie de } \\
\text { preguntas sobre los contenidos }\end{array}$ \\
\cline { 2 - 3 } & Material imprimible & Recortables de personajes para imprimir y colorear \\
M7 & $\begin{array}{l}\text { Mural: mural decorativo en el que se combina un dibujo con cada una de las } \\
\text { cinco vocales }\end{array}$ \\
& Tarjetas: 70 tarjetas que constituyen el método de lectoescritura \\
Material imprimible & $\begin{array}{l}\text { Mural: mural decorativo en el que se combina un dibujo con cada una de las } \\
\text { cinco vocales } \\
\text { Tarjetas: } 70 \text { tarjetas que constituyen el método de lectoescritura }\end{array}$ \\
\hline
\end{tabular}


Al igual que en el caso de los roles docentes y discentes, podemos hablar de los roles que promueven los materiales complementarios. En el caso concreto de los materiales señalados, M3, M4 y M6 podemos decir que se asocian con un modelo mixto si bien, el uso que se haga de ellos en el aula condicionará su tendencia hacia un rol pasivo o activo.

\section{DISCUSIÓN Y CONCLUSIONES}

A la vista de los resultados descritos, una conclusión común al análisis de las tres variables que nos han servido para determinar el modelo pedagógico (actividades, roles y recursos complementarios) es que, en gran medida, el modelo pedagógico dependerá más del rol que adopte el profesorado y el uso que haga del mismo que, del modelo que a priori se infiere de dicho material (Trueba, 1997). Para ilustrar esta situación señalamos, por ejemplo, que el trabajo cooperativo o en grupo no se refleja de manera explícita en ninguno de los materiales si bien, el docente podrá utilizar esta estrategia organizativa a la hora de usar el ODA.

Con respecto a las actividades, parece que los materiales analizados no apuntan evidencias de que el aporte tecnológico ayude a promover aprendizajes más exigentes ni perspectivas pedagógicas más comprensivas, globalizadas y capaces de adaptarse a la diversidad de estilos cognitivos que puedan encontrarse en el aula. Esto ya ha sido apuntado en investigaciones como la de Area (2015) en la que se pone de manifiesto que, para pasar del paradigma reproductivo del libro de texto a un modelo pedagógico más innovador, se debe reinventar el material didáctico más allá del soporte en el que se presente.

Siguen predominando las tareas de memorización, incluso en aquellos materiales diseñados específicamente como MDD para ser utilizados en el entorno escolar, siendo más anecdótica la presencia de tareas que inviten a la reflexión, a la comprensión o creación. Precisamente, la posibilidad de promover estos niveles más exigentes cognitivamente se puede inferir en mayor medida en los ODA analizados, si bien dependeríamos siempre de que el enfoque docente acompañe en esta dirección.

Es importante, asimismo, que los MDD cuenten con diversas actividades que puedan abarcar las diferentes fases de una secuencia didáctica, conscientes de que carecen de un pilar fundamental como son las actividades para abordar los conocimientos previos del alumnado, tan importantes para el desarrollo de la comprensión. Sin embargo, como punto débil está la tendencia a esta estructura lineal que se manifiesta tanto a la hora de organizar los tipos de actividades dentro de la secuencia didáctica, que siempre es la misma (introducción, desarrollo, extensión o refuerzo, y evaluación), como de la lógica organizativa de las actividades que componen la fase de desarrollo dentro de la propia secuencia didáctica. Estas actividades siempre van de lo simple a lo complejo o de la teoría a la aplicación.

Parece que, tras los análisis realizados, no se puede constatar que las potencialidades tecnológicas de un material didáctico digital no sirvieron para romper de alguna manera con este tipo de racionalidad más cercana al pensamiento deductivo y más alejada de otras formas de pensamiento, como puede ser el inductivo.

Por otro lado, el uso de ODA podría de nuevo facilitar la ruptura de esta tendencia predominante y facilitar el ajuste a los diferentes estilos de aprendizaje que puedan estar presentes en el aula. Qué duda cabe que para eso necesitamos la competencia docente en este sentido, aspecto que se señala en la literatura existente (Gonçalves, \& Almeida, 2016).

En relación con los roles, los datos sugieren que los roles docentes y discentes tienden a la pasividad. No obstante, en el caso de los roles que los materiales promueven en el alumnado, se dan casos mixtos, aun ten- 
diendo a la pasividad. Sin embargo, entendemos que el rol del alumnado en buena parte dependerá del rol que adopte el profesorado y de cómo éste enfoque el uso de tal material (de forma individual o en pareja/ grupos, con más o menos poder de decisión, etc.). A esta conclusión también llegó el equipo investigador en las fichas de análisis del proyecto en el que se enmarca el presente artículo. Se muestra a continuación un ejemplo: "al tratarse de un álbum ilustrado digitalizado, es complicado clasificarlo dentro de un modelo pedagógico determinado. Ayuda en el proceso de aprendizaje a través de aspectos como la atención, la motivación, escucha, expresión oral..., no obstante, los papeles del docente y estudiante, así como la pedagogía implícita depende notablemente del uso que el adulto le dé" (M5).

Por último, observamos que los recursos complementarios son prácticamente nulos en los materiales examinados. Sorprende que no se aprovechen las potencialidades de las tecnologías digitales para incorporar otros recursos multimedia, portafolios, vídeos o, por ejemplo, el acceso a glosarios online, y que, sin embargo, siempre se encuentren materiales imprimirles listos para usar en el aula.

En general, se echan en falta guías de uso y guías didácticas dónde se dé cuenta de la lógica que organiza los contenidos, el modelo pedagógico del material, o de cómo adaptarlo a la diversidad existente en las aulas.

Por otro lado, se debe destacar que en ningún caso estos recursos ayudan a atender a la diversidad del aula, a plantear los contenidos de una forma globalizada o que acerquen al alumnado a su realidad más próxima. Esto es sorprendente dado que a todos estos aspectos se les otorga una importancia primordial en el Decreto 330/2009.

A la vista de lo analizado, parece que los diseños de los materiales didácticos digitales proporcionados por las editoriales para la etapa de El no logran, por el momento, contribuir a superar los modelos pedagógicos tradicionales, tal y como afirmaban Ricaurte y Carli (2016) o Selwyn (2016). Igualmente, se ha constatado que estos no aprovechan las potencialidades del medio digital para el que fueron creados, siendo todavía muy similares o sus homólogos en papel (Area, 2019; Area, \& Rodríguez, 2017; Rodríguez, 2019).

Cerramos la discusión destacando que, a la vista de lo expuesto, todavía queda camino por recorrer para que los materiales didácticos digitales cumplan con las exigencias de versatilidad, diversidad y significatividad que demanda la normativa educativa gallega, así como la metamorfosis (Area, 2017) necesaria para la incorporación efectiva de estos "nuevos” materiales.

En cuanto a las limitaciones del presente estudio, contemplamos las dificultades de acceso a la muestra al ser MDD de acceso restringido para usuarios registrados. Respecto a las líneas de trabajo futuras, será interesante seguir profundizando en otros elementos que contribuyan a describir los modelos pedagógicos como el tipo de contenidos o el enfoque de evaluación. Es igualmente importante estudios que analicen el uso de los MDD en esta etapa educativa.

\section{FINANCIAMIENTO}

Este artículo se ha realizado en el marco del proyecto de investigación titulado "Los materiales didácticos digitales en la Educación Infantil. Análisis y propuestas para su uso en la escuela y elhogar (RTI2018093397-B-I00)", financiado por el Fondo Europeo de Desarrollo Regional (FEDER), el Ministerio de Ciencia e Innovación y la Agencia Estatal de Investigación (AEI). 


\section{REFERENCIAS}

Alba, C. (2012) Aportaciones del Diseño Universal para el Aprendizaje y de los materiales digitales en el logro de una enseñanza accesible. En J. Navarro, M. T.; Fernández, F.J. Soto \& F. Tortosa (Coords.), Respuestas flexibles en contextos educativos diversos (pp. 1-13). Consejería de Educación, Formación y Empleo.

Area, M. (1994). Los medios y materiales impresos en el currícu-

lum. En M. Sancho (Coord), Para una tecnología educativa (pp. 85-114). Horsori.

Area, M. (2015). La escuela en la encrucijada de la sociedad digital. Cuadernos de pedagogía, 462, 26-31.

Area, M. (2017). La metamorfosis digital del material didáctico tras el paréntesis Gutenberg. Revista Latinoamericana de Tecnología Educativa, 16(2), 13-28.

Area, M. (2019). Informe sobre las plataformas y materiales didácticos digitales en la Comunidad Autónoma de Canarias: estudio I. EDULLAB. Laboratorio de Educación y Nuevas Tecnologías de la Universidad de La Laguna. https://riull.ull.es/ xmlui/handle/915/16118

Area, M., \& Rodríguez, J. (2017). De los libros de texto a los materiales didácticos digitales. RELATEC: Revista Latinoamericana de Tecnología Educativa, 16(2), 9-12.

Bonafé, J. M. (2002). Políticas sobre el libro de texto escolar. Ediciones Morata.

Cañas, A. M. (2010). Los materiales en educación infantil. Revista digital Innovación y experiencias educativas, (27), 1-9.

Decreto 330/2009, de 4 de junio, por el que se establece el currículo de la educación infantil en la Comunidad Autónoma de Galicia. Diario Oficial de Galicia, 121, 10773-10799. https:// politicasocial.xunta.gal/sites/w polso/files/arquivos/normativa/decreto 3302009 cas.pdf

Daly, L., \& Beloglovsky, M. (2016). Loose parts 2: Inspiring play with infants and toddlers. Redleaf Press.

Doyle, W. (1983). Academic work. Review of Educational Research, 53(2), 159-199.

Elliot, J. (1990). La investigación-acción en educación. Morata.
Gabarda, V., Marín, D., \& Romero, M.M. (2021). Evaluación de recursos digitales para población infantil. EDMETIC, Revista de Educación Mediática y TIC, 10(1), 135-153. https://doi. org/10.21071/edmetic.v10i1.13125

Gimeno, J. (1988). El currículo: una reflexión sobre la práctica. Morata

Gonçalves, D., \& Almeida, S. (2016). Learning and teaching using digital books: opportunities and constraints. Revista profesorado, 20(1), 49-60. http://recyt.fecyt.es/index.php/profesorado/article/view/49867

Liébana, J., Romero, R., \& Ibáñez, M. (2001). La evaluación de materiales didácticos para la educación a distancia. RIED. Revista Iberoamericana de Educación a Distancia, 4(1), 73-96. https://doi.org/10.5944/ried.4.1.1192

Losada, C., \& Rodríguez, J. (2019). Análisis del proyecto de educación digital (E-DIXGAL): la visión del profesorado de educación primaria. Digital Education Review, 36, 171-189. https:// doi.org/10.1344/der.2019.36.171-189

Martínez, M. E., \& Fernández, I. (2020). El diseño universal de aprendizaje para la creación de contenidos digitales inclusivos. En M. Raposo \& M. Cebrián (2020) Tecnologías para la formación de educadores en la sociedad del conocimiento (pp. 125-136). Pirámide.

Moreno, F. M. (2017). La Influencia de los Materiales Manipulativos durante el Proceso de Enseñanza/Aprendizaje en Segundo Ciclo de Educación Infantil [Tesis doctoral]. Universidad de Murcia, Murcia, España. https://digitum.um.es/digitum/ bitstream/10201/53424/1/Fco.\%20Manuel\%20Moreno\%20 Lucas\%20Tesis\%20Doctoral\%20sin\%20Art.pdf

Nicholson, S. (1973). The Theory of Loose Parts.Man/Society/Technology. A Journal of Industrial Arts Education, 32(4), 172-75.

Peirats, J., Gallardo, I. M., San Martín, A., \& Waliño, M. J. (2016). Análisis de la industria editorial y protocolo para la selección del libro de texto en formato digital. Profesorado, Revista de currículum y formación del profesorado, 20(1), 75-89. https:// recyt.fecyt.es/index.php/profesorado/article/view/49869 
Ricaurte-Quijano, P., \& Carli-Álvarez, A. (2016). El proyecto Wiki Learning: Wikipedia como entorno de aprendizaje abierto. Comunicar, 24(49), 61-69. https://doi.org/10.3916/C49$\underline{2016-06}$

Rodríguez, J. (2000). Os materiais curriculares impresos e a reforma educativa en Galicia (tesis doctoral), Universidade de Santiago de Compostela, Santiago de Compostela, España. Rodríguez, N. (2019). Los libros de texto digitales. Un análisis descriptivo e interpretativo de sus características. (Tesis doctoral). Universidad de Santiago de Compostela, Santiago de Compostela, España. http://hdl.handle.net/10347/23309

Sanabria, A. L., Álvarez, Q., \& Peirats, J. (2017). Las políticas educativas en la producción y distribución de materiales didácticos digitales. Revista Latinoamericana de Tecnología Educativa, 16(2), 63-77. https://relatec.unex.es/article/ view/3063

Sánchez, J.M., \& Arathoon, A.I. (2017) Recursos digitales y Diseño Universal para el Aprendizaje. En C. Pastor (coord.) Diseño universal para el aprendizaje: educación para todos y prácticas de enseñanza inclusivas (pp. 89-122). Morata.

Selwyn, N. (2016). Is technology good for education? John Wiley \& Sons.

Trueba, B. (1997). Modelos didácticos y materiales curriculares en educación infantil. Investigación en la Escuela, 33, 3646. https://revistascientificas.us.es/index.php/IE/article/ view/7931/7023

Zabalza, M.A., \& Zabalza, M. (2012). La docencia en un mundo en cambio. Algunos dilemas básicos de la acción docente. En M. A. Zabalza \& M. Zabalza (Coords.), Profesores y profesión docente (pp. 25-43). Morata. 